International Mathematical Forum, Vol. 8, 2013, no. 14, 697 - 700 HIKARI Ltd, www.m-hikari.com

\title{
A Note on Additive Mappings and Commutative Conditions for Prime Rings
}

\author{
Francesco Rania
}

Department of Legal, Historical, Economic and Social Sciences Magna Graecia University of Catanzaro, Campus loc. Germaneto

Viale Europa, 88100 Catanzaro, Italy

raniaf@unicz.it

Copyright (c) 2013 Francesco Rania. This is an open access article distributed under the Creative Commons Attribution License, which permits unrestricted use, distribution, and reproduction in any medium, provided the original work is properly cited.

\begin{abstract}
Let $R$ be a prime ring of characteristic different from $2, f: R \rightarrow R$ a non-zero additive mapping on $R$, such that $f(x y)=f(x) y+f(y) x$. We prove that if $[f(x), f(y)]=0$ for all $x, y \in R$, then $R$ must be commutative.
\end{abstract}

\section{Mathematics Subject Classification: 16N60}

Keywords: Prime rings, additive mappings

\section{Introduction}

In this paper we study a prime ring $R$ with an additive mapping which satisfies a commutativity condition on $R$. More precisely, let $R$ be a prime ring with center $Z(R)$ and let $S$ be a subset of $R$, we say that a mapping $F: R \rightarrow R$ is commuting on $S$ if $[F(x), x]=0$, for any $x \in S$, moreover the map $F$ is said to be centralizing if $[F(x), x] \in Z(R)$, for any $x \in S$. In all that follows we will denote by $C$ the extended centroid on $R$. $C$ is the center of the Martindale quotients ring of $R$. All that we need about $C$ is that it is a field, under the assumption that $R$ is a prime ring. Moreover $a x=x a$, for all $x \in R, a \in C$. In [5] Posner proved that $R$ is commutative if it admits a non-zero centralizing derivation. This well known result was the starting point 
of a number of papers concerning the study of such mappings. One might wonder what can be said about the relationship between an additive map $F: R \rightarrow R$ and a derivation $d$ of $R$, such that $q(x)=[d(x), F(x)] \in Z(R)$, for all $x$ in a suitable subset $S$ of $R$. When $F$ is a derivation we say that $q(x)$ is a quadratic central differential identity on $S$. The study of such kind of identities of prime rings was given by Lanski. In [3] he showed that if $d$ and $\delta$ are non-zero derivations on $R$, such that $[d(x), \delta(x)] \in Z(R)$, for all $x \in R$, then either there exists $\lambda$ in the extended centroid $C$ of $R$ such that $d=\lambda \delta$ or $\operatorname{char}(R)=2$ and $R$ satisfies $s_{4}\left(x_{1}, \ldots, x_{4}\right)$, the standard identity of degree 4 . The same conclusion holds when $x$ belongs in a noncentral Lie ideal of $R([2])$. A result of similar flavour has been obtained by Lee in [4]. He studied the case when $[d(x), \delta(x)] \in Z(R)$, for any $x \in \varrho$, a non-zero right ideal of $R$. He proved that, under this assumption, either there exists $\lambda \in C$ such that $d=\lambda \delta$ or $d(\varrho) \varrho=\delta(\varrho) \varrho=0$, unless $\operatorname{char}(R)=2$ and $\varrho$ satisfies the identity $s_{4}\left(x_{1}, \ldots, x_{4}\right) x_{5}$. More recentely Beidar, Bresar and Chebotar obtained a definitive result on the functional identity $[d(x), F(x)]=0$, for all $x \in R$, where $F$ is an additive map on $R$ and $d$ is a derivation of $R$. In case $R$ has characteristic different from 2, they proved that there exist $\lambda \in C$ and an additive map $\mu: R \rightarrow C$, such that $F(x)=\lambda d(x)+\mu(x)$, for any $x \in R[1]$.

This paper is motived by the previous cited results. More precisely we will prove the following results:

Theorem 1.1 Let $R$ be a prime ring of characteristic different from 2, $f: R \rightarrow R$ a non-zero additive mapping on $R$, such that $f(x y)=f(x) y+f(y) x$. We prove that if $[f(x), f(y)]=0$ for all $x, y \in R$, then $R$ is commutative.

\section{Main Results}

In all that follows let $R$ be a prime ring and $f: R \rightarrow R$ be a non-zero additive mapping on $R$, such that $f(x y)=f(x) y+f(y) x$ for all $x, y \in R$. We begin with the following useful Lemma:

Lemma 2.1 $R$ does not contain any non-zero square-zero element.

Proof. Suppose that there exists $0 \neq a \in R$ such that $a^{2}=0$. Denote $I=\{y \in R: y a=0\} . \quad I$ is a non-zero left ideal of $R$. For all $y \in I$, $0=f(y a)=f(y) a+f(a) y$. Thus, for any $r \in R$

$$
0=f(r y) a+f(a) r y=f(y) r a+f(a) r y .
$$

Replace in (1) $r$ by $r y, x \in I$, and get $f(a) r x y=0$, which is either $f(a)=0$ or $x y=0$ for any $x, y \in I$. If $f(a)=0$, from (1) we have $f(y)=0$, for all $y \in I$, and so $(0)=f(R I)=f(R) I$, that is $f(R)=(0)$, a contradiction. 
Let $x y=0$, for all $x, y \in I$. Again from (1), by right multiplying by $x \in I$, $0=f(r y) a x=f(y) r a x$, that is either $f(y)=0$ or $a x=0$, for all $x \in I$. In the first case, as above, we have the contradiction $f(r)=(0)$. In the second one, since $I$ is a non-zero left ideal, we get that contradiction $a=0$.

Therefore $R$ does not contain any non-zero square-zero element.

Lemma 2.2 If $[f(x), x]=0$ for all $x \in I$, then $R$ is commutative.

Proof. Suppose by contradiction that $R$ is not commutative. Let $x, y, z$ any elements of $R$. By our assumption we have that

$$
\begin{aligned}
0 & =f([x, y] z)=f([x, y]) z+f(z)[x, y]=f(x y-y x) z+f(z)[x, y]= \\
& =f(x) y+f(y) x-f(y) x-f(x) y+f(z)[x, y]=f(z)[x, y] .
\end{aligned}
$$

Thus, for any $r \in R, f(z)[x r, y]=0$ that is $f(z) x[r, y]=0$. In other words it follows that $f(R) R[R, R]=(0)$. Since $R$ is prime, it follows that either $[R, R]=(0)$ or $f(R)=(0)$. In any case we have a contradiction, since we suppose $f \neq 0$ and $R$ non-commutative.

Theorem 2.3 If $[f(x), f(y)]=0$ for all $x, y \in R$, then $R$ is commutative.

Proof. Let $x, y \in R$, then

$$
0=[f(x y), f(y)]=[f(x) y+f(y) x, f(y)]=f(x)[y, f(y)]+f(y)[x, f(y)] .
$$

Replace $x$ with $x r$ in (2):

$$
\begin{aligned}
0 & =(f(x) r+f(r) x)[y, f(y)]+f(y)[x r, f(y)]= \\
& =f(x) r[y, f(y)]+f(r) x[y, f(y)]+f(y)[x, f(y)] r+f(y) x[r, f(y)] .
\end{aligned}
$$

Moreover, since $f(y)[x, f(y)]=-f(x)[y, f(y)]$, it follows that

$$
f(x) r[y, f(y)]+f(r) x[y, f(y)]-f(x)[y, f(y)] r+f(y) x[r, f(y)]=0 .
$$

In particular, choose $z \in R$ and substitute $y=f(z)$. As a consequence we get: $f^{2}(z) x\left[r, f^{2}(z)\right]=0 \quad \forall x, r, z \in R$. Thus either $f^{2}(z)=0$ or $f^{2}(z) \in Z(R)$, the center of $R$.

Suppose there exists $z_{1} \in R$ such that $0 \neq f^{2}\left(z_{1}\right) \in Z(R)$. Replace in (3) $x=f\left(z_{1}\right)$ and obtain $f^{2}\left(z_{1}\right)[y, f(y)]=0 \quad \forall y \in R$. Since $R$ is prime, $Z(R)$ cannot contain any zero-divisor element, then $[y, f(y)]=0$. In other words we have two cases: either $f^{2}(x)=0$, for all $x \in R$, or $[y, f(y)]=0$, for all $y \in R$.

Suppose that $f^{2}(x)=0$, for all $x \in R$. In particular $0=f(f(x y))=$ $f(y) f(x)+f(x) f(y)=2 f(x) f(y)$. Since $\operatorname{char}(R) \neq 2$, we have $f(x) f(y)=0$ for all $x, y \in R$ and by Lemma 2.1, since $R$ cannot contain non-zero squarezero elements, we have that $f(x)=0$, that is $f(R)=(0)$, a contradiction. Thus $R$ must satisfy $[x, f(x)]=0$ and we conclude, by Lemma 2.2 , that $R$ is commutative. 


\section{References}

[1] K.I. Beidar, M. Bresar, M.A. Chebotar, Functional identities with rindepen-dent coefficients, Comm. Algebra, 30 (12) (2002), 5725-5755.

[2] C. Lanski, Differential identities of prime rings, Kharchenko's theorem and applications, Contemp. Math., 124 (1992), 111-128.

[3] C. Lanski, Quadratic central differential identities of prime rings, Nova J. Algebra Geom., 1 (2) (1992), 185-206.

[4] T.K. Lee, Derivations and centralizing mappings in prime rings, Taiwanese J. of Math., 1 (3) (1997), 333-342.

[5] E.C. Posner, Derivations in prime rings, Proc. Amer. Math. Soc., 8 (1957), 1093-1100.

Received: January 23, 2013 